\title{
Erratum: Time-Dependent Correlations in an Inhomogeneous One-Component Plasma ${ }^{1}$
}

\author{
B. Jancovici, J. L. Lebowitz, and Ph. A. Martin
}

Received November 7, 1994

The Section Wedge and Appendix A are erroneous and must be deleted. The mistake comes from the assumption following Eq. (A.5). It can be seen in the static case that this assumption is not satisfied by the correct correlation function derived by Choquard et al. ${ }^{(1)}$ and confirmed by Jancovici. ${ }^{(2)}$

\section{REFERENCES}

1. Ph. Choquard, B. Piller, R. Rentsch, and P. Vieillefosse, J. Stat. Phys. 55:1185 (1989).

2. B. Jancovici, J. Stat. Phys., to appear, 80 (1995).

'This paper originally appeared in J. Stat. Phys. $41: 941$ (1985). 